\title{
The Role of 18-Fluorodeoxyglucose Positron Emission Tomography/Computed Tomography (FDG-PET/CT) in Management of Nocardiosis: A Retrospective Study and Review of the Literature
}

\author{
Ili Margalit (D) - Anat Yahav • Yaara Ben Ari · Haim Ben-Zvi · Avivit Shoham • Elad Goldberg • \\ Michal Weiler-Sagie $\cdot$ Noam Tau $\cdot$ Khitam Muhsen · Jihad Bishara · Mical Paul Hanna Bernstine \\ Dafna Yahav
}

Received: April 28, 2021 / Accepted: June 30, 2021 / Published online: July 20, 2021

(C) The Author(s) 2021

\section{ABSTRACT}

Introduction: 18-Fluorodeoxyglucose positron emission tomography/computed tomography (FDG-PET/CT) is a well-established tool for managing metastatic infections. Nocardiosis, a

Hanna Bernstine and Dafna Yahav equally contributed to this article.

I. Margalit $(\varangle) \cdot$ J. Bishara $\cdot$ D. Yahav

Infectious Diseases Unit, Rabin Medical Center, Beilinson Hospital, 39 Jabotinsky Road, Petah Tikva, Israel

e-mail: ilimargalit@gmail.com

I. Margalit · Y. Ben Ari · E. Goldberg · N. Tau .

J. Bishara $\cdot$ H. Bernstine $\cdot$ D. Yahav

Sackler Faculty of Medicine, Tel Aviv University,

Ramat Aviv, Tel Aviv-Yafo, Israel

A. Yahav

Radiology Institute, Rambam Health Care Campus, Haifa, Israel

H. Ben-Zvi

Microbiology Laboratory, Rabin Medical Center, Beilinson Hospital, Petah Tikva, Israel

\section{A. Shoham}

Department of Diagnostic Imaging, Rabin Medical Center, Beilinson Hospital, Petah Tikva, Israel

\section{E. Goldberg}

Department of Internal Medicine F, Rabin Medical Center, Beilinson Hospital, Petah Tikva, Israel primarily pulmonary infection, disseminates at high rates. Routine imaging includes chest CT and brain imaging. We examined the use of FDG-PET/CT in nocardiosis and assessed its contribution to diagnosis and management.

Methods: A retrospective study in two tertiary medical centers during 2011-2020. Individuals with nocardiosis for whom FDG-PET/CT was implemented for any reason were included and

M. Weiler-Sagie

Nuclear Medicine Department, Rambam Health

Care Campus, Haifa, Israel

M. Weiler-Sagie · M. Paul

Rappaport Faculty of Medicine, Technion, Israel Institute of Technology, Haifa, Israel

N. Tau

Department of Diagnostic Imaging, Sheba Medical Center, Ramat Gan, Israel

K. Muhsen

Department of Epidemiology and Preventive Medicine, Sackler Faculty of Medicine, School of Public Health, Tel Aviv University, Ramat Aviv, Tel Aviv-Yafo, Israel

M. Paul

Infectious Diseases Institute, Rambam Health Care Campus, Haifa, Israel

H. Bernstine

Nuclear Medicine Institute, Rabin Medical Center, Beilinson Hospital, Petah Tikva, Israel 
their medical records were reviewed. A boardcertified nuclear medicine physician independently reviewed all scans. Additionally, a systematic review was conducted according to the PRISMA guidelines, to extract data from publications reporting FDG-PET/CT use for the management of nocardiosis.

Results: FDG-PET/CT contributed to the management of all seven patients who met inclusion criteria. It assisted in ruling out an underlying malignancy $(29 \%, 2 / 7)$; establishing a wide infection extent $(57 \%, 4 / 7)$; and affecting decisions regarding treatment $(57 \%, 4 / 7)$, including drug regimen, oral step-down, and duration of therapy. We identified 20 published case reports on this topic. In $80 \%(16 / 20)$, FDG-PET/CT contributed to the management of nocardiosis similar to our study. In addition, in most of the literature cases, FDG-PET/CT guided the diagnostic biopsy. Conclusion: FDG-PET/CT is valuable in the diagnosis and management of individuals with nocardiosis. The contribution of incorporating FDG-PET/CT to the management of individuals with nocardiosis and its role in monitoring treatment response and shortening treatment duration should be evaluated in prospective studies.

Keywords: Imaging; Nocardia infections; Opportunistic infections

\section{Key Summary Points}

\section{Why carry out this study?}

Nocardiosis is a rare infection that may be life-threatening; however, the evidence for its management is scarce and mostly based on expert opinion.

Limited data are available to guide initial evaluation of infection extent, as well as duration of treatment.

In this study we evaluated the contribution of PET/CT in the management of individuals with nocardiosis, in two tertiary healthcare centers. In addition, we systematically reviewed the medical literature and gathered the existing evidence on this subject.

\section{What was learned from the study?}

Incorporating PET/CT can facilitate the diagnosis of nocardiosis by revealing unknown extrapulmonary dissemination, excluding an underlying malignancy and guiding biopsy.

Incorporating PET/CT can assist in treatment-related decisions, e.g., promote shortening of the total antimicrobial therapy duration, rule out nocardiosis relapse in the setting of breakthrough fever, etc.

\section{INTRODUCTION}

Integrated positron emission tomography/computed tomography (PET/CT) with 18-fluorodeoxyglucose (FDG) is a useful modality for managing some infections [1], mostly disseminated infections [2, 3]. FDG-PET/ CT can predict extracardiac infective endocarditis (IE) complications [4], and contributes to its treatment approach and decision-making $[5,6]$. FDG-PET/CT has therefore become an established tool in the management of IE, and was incorporated into its diagnostic criteria [7].

FDG-PET/CT is also useful in the management of musculoskeletal infections [8], and may be particularly effective in the work-up of opportunistic infections. It has been used as an adjunct diagnostic method for Pneumocystis jirovecii [9], and for predicting therapy response in individuals with tuberculosis [10]. Similarly, it seems useful for assessment of both the initial systemic spread and treatment response in cases of invasive fungal infections [11].

Nocardiosis, a pulmonary opportunistic infection which disseminates in up to $43 \%$ of cases [12], usually occurs in organ transplant recipients [12], individuals subjected to corticosteroid therapy for any reason [13], and individuals with chronic obstructive pulmonary disease [14] or cancer [15]. Accordingly, FDGPET/CT may be useful both in diagnosing nocardiosis dissemination as well as revealing an underlying malignancy. Revealing the extent 
of the infection may assist in management decisions such as need for invasive percutaneous procedures or surgical interventions. In addition, considering the prolonged antimicrobial course usually administered for nocardiosis [16], FDG-PET/CT can have a role in guiding treatment duration.

The aim of the current study was to describe the uses of FDG-PET/CT in the management of individuals with nocardiosis, and suggest future directions. The core question of this study was whether FDG-PET/CT influenced the management of individuals with nocardiosis.

\section{METHODS}

We describe a case series and systematic review.

For the case series, we reviewed the medical charts of all individuals with Nocardia infection who had undergone a FDG-PET/CT examination in two large volume tertiary medical centers between January 2011 and December 2020.

Patients with a positive culture for Nocardia spp. from any source were identified using the microbiology laboratory computerized database in each hospital. We included consecutive adult patients from whom the isolated Nocardia was considered to represent an infection (nocardiosis) rather than asymptomatic colonization [17], and were treated accordingly. The medical records of these individuals were further reviewed to identify patients for whom FDGPET/CT was implemented as part of their diagnostic work-up and/or follow-up. FDG-PET/CT scans conducted for other purposes were allowed if they occurred within the pre-diagnosis symptomatic period (up to 1 month) or during the 6 months following the diagnosis of nocardiosis. Patients' demographics, comorbidities, and clinical details regarding their $\mathrm{No}$ cardia infection, including C-reactive protein level (CRP), imaging and microbiological data, were collected. In order to define the contribution of FDG-PET/CT to the management of our patients, we considered for each case whether the conduction of FDG-PET/CT prompted the diagnosis, ruled out alternative diagnoses and underlying diseases, or guided treatment and management decision-making.

\section{PET/CT Implementation Protocol}

After fasting for at least $4 \mathrm{~h}$, all patients underwent a FDG-PET/CT scan, executed by a GE Discovery STE Whole Body PET/CT scanner (GE Medical Systems, Milwaukee, WI, USA) or similar. CT images were acquired at $120 \mathrm{kV}$ and $80 \mathrm{~mA}$, pitch $1.75,0.8 \mathrm{~s}$ per tube rotation, and slice thickness of $3.75 \mathrm{~mm}$. During the CT examination, $80-120 \mathrm{~mL}$ of a contrast agent (Ultravist 300, Schering AG, Berlin, Germany) was intravenously administered to ensure fully diagnostic CT data (if there was no contraindication). The PET scan was performed 60-75 min after an ${ }^{18} \mathrm{~F}$-FDG intravenous injection of 7-10 mCi (depending on the individual's weight). The contrast-enhanced CT was used also for attenuation correction of the PET data. The PET scan was performed from the base of the skull to the mid-thigh, $1.5-2 \mathrm{~min}$ per bed position, resulting in a total scan time of approximately $15-20 \mathrm{~min}$.

For the purpose of the current study, a boardcertified nuclear medicine physician (HB) with 15 years of experience independently reviewed all FDG-PET/CT scans from both centers, confirmed the results, and measured the lesion's size, volume, and standard uptake volume (SUV).

\section{Statistical Analysis}

The statistical methods used in this study were mainly descriptive. The correlation between CRP and maximum SUV was implemented using Spearman's rank correlation coefficient.

\section{Ethical Approval}

The ethics committees of Rabin Medical Center and Rambam Health Care Campus approved the study protocol. An exemption from informed consent was given by the ethics committees because of the retrospective design of the study. All methods were performed in accordance with the Helsinki Declaration of 1964 and its later amendments. 


\section{Systematic Review}

We reviewed the medical literature for publications reporting the use of FDG-PET/CT for the management of individual with nocardiosis. Two reviewers (IM and DY) independently searched the literature and retrieved all English written study types reporting in detail the use of FDG-PET/CT as part of the diagnosis or management of individuals with nocardiosis. The search was conducted in the MEDLINE database, Cochrane library, and ECCMID library, combining the following search terms: Nocardia or Nocardiosis and PET or Positron emission or FDG or Fluorodeoxyglucose or Nuclear medicine or Mimicking malignancy. The contribution of FDG-PET/CT to case management was assessed as above.

\section{RESULTS}

Nocardia was isolated from 131 individuals in both centers between January 2011 and December 2020. Of these, 7 (5.3\%) met inclusion criteria.

Characteristics, clinical scenario and imaging findings of the seven patients included in the study are presented in Table 1 . Of these, $71 \%(5 / 7)$ were male, the average age was 63.7 (range 43-86; standard deviation [SD] 14.2) years, and $71 \%(5 / 7)$ were subjected to immune suppressive conditions (29\% [2/7] solid organ transplant recipients; 43\% [3/7] hematological malignancies). The Nocardia isolated from $86 \%$ $(6 / 7)$ underwent species identification using polymerase chain reaction (PCR). Of these, $83 \%$ $(5 / 6)$ were identified as Nocardia cyriacigeorgica and $17 \%(1 / 6)$ were identified as Nocardia farcinica. The Nocardia isolated from the remaining patient was diagnosed using mass spectrometry, and species level was not appropriately determined.

The contributions of FDG-PET/CT performed around the diagnosis included proof of clinically significant infection in all included cases, demonstration of previously unknown extrapulmonary dissemination $(57 \%, 4 / 7)$, exclusion of an underlying malignancy $(29 \%, 2 / 7)$ or disease progression $(29 \%, 2 / 7)$, and assistance in treatment-related decisions $(57 \%, 4 / 7)$ (Table 1$)$.

FDG-PET/CT revealed patchy colonic uptake (Figs. 1 and 2) that was presumed to reflect nocardiosis involvement, although this remained questionable in the absence of microbiological evidence. In one of these patients (patient 3), detecting the colonic involvement (colonic malignancy is unlikely since the patient was cured following appropriate antimicrobial therapy) redefined the disease as disseminated infection, rather than local pulmonary infection (Fig. 1). In another patient (patient 6), lower limb lymphocutaneous involvement, which was not detected on physical examination, was revealed by FDGPET/CT. In an additional patient (patient 7), FDG-PET/CT revealed a cardiac abscess representing prosthetic aortic valve endocarditis, which was not detected by transthoracic echocardiography, and helped to rule out progression of the underlying hematologic malignancy (Fig. 3).

In one immunocompromised patient (patient 1), resolution of the pulmonary infectious process was demonstrated on FDG-PET/CT performed approximately 6 weeks following the initiation of antimicrobial therapy. This supported antibiotic monotherapy for a period of 6 months, the shortest treatment duration acceptable for stable pulmonary disease in immunocompromised individuals, at that time [18]. This patient was considered cured from nocardiosis as was evident during follow-up visits up to 1 year following treatment completion.

In another patient (patient 2) with breakthrough fever during treatment for nocardiosis, FDG-PET/CT demonstrated progression of the underlying malignancy. Accordingly, nocardiosis relapse was ruled out and the anti-Nocardia regimen remained unchanged. In an additional patient (patient 6), FDG-PET/CT demonstrated active pulmonary nodules and cutaneous involvement and proved that the positive blood culture reflected nocardiosis and not contamination, as initially assumed. Consequently, systemic therapy was started. In an additional patient (patient 7), the detection of cardiac abscess which was not revealed by 


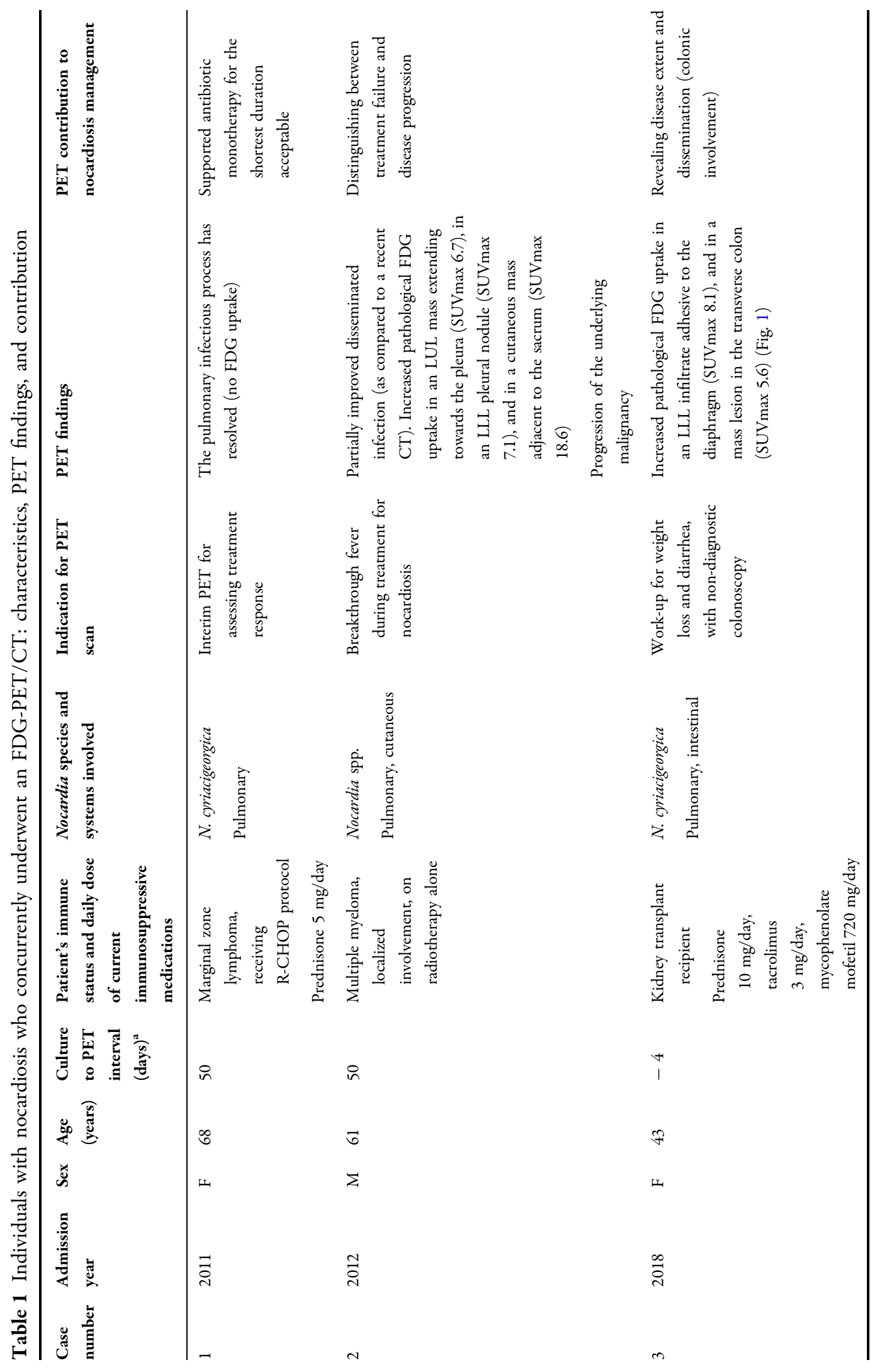




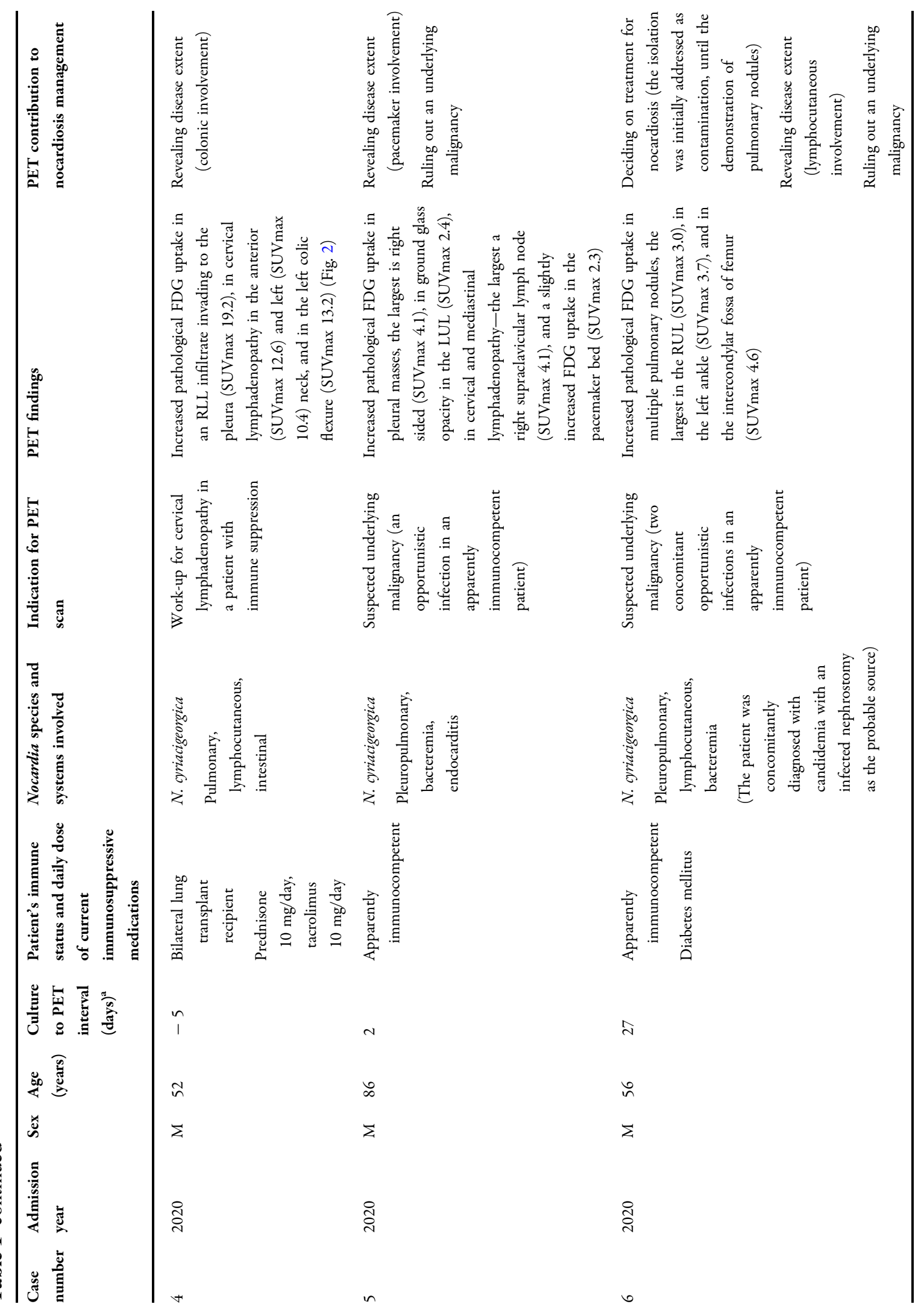




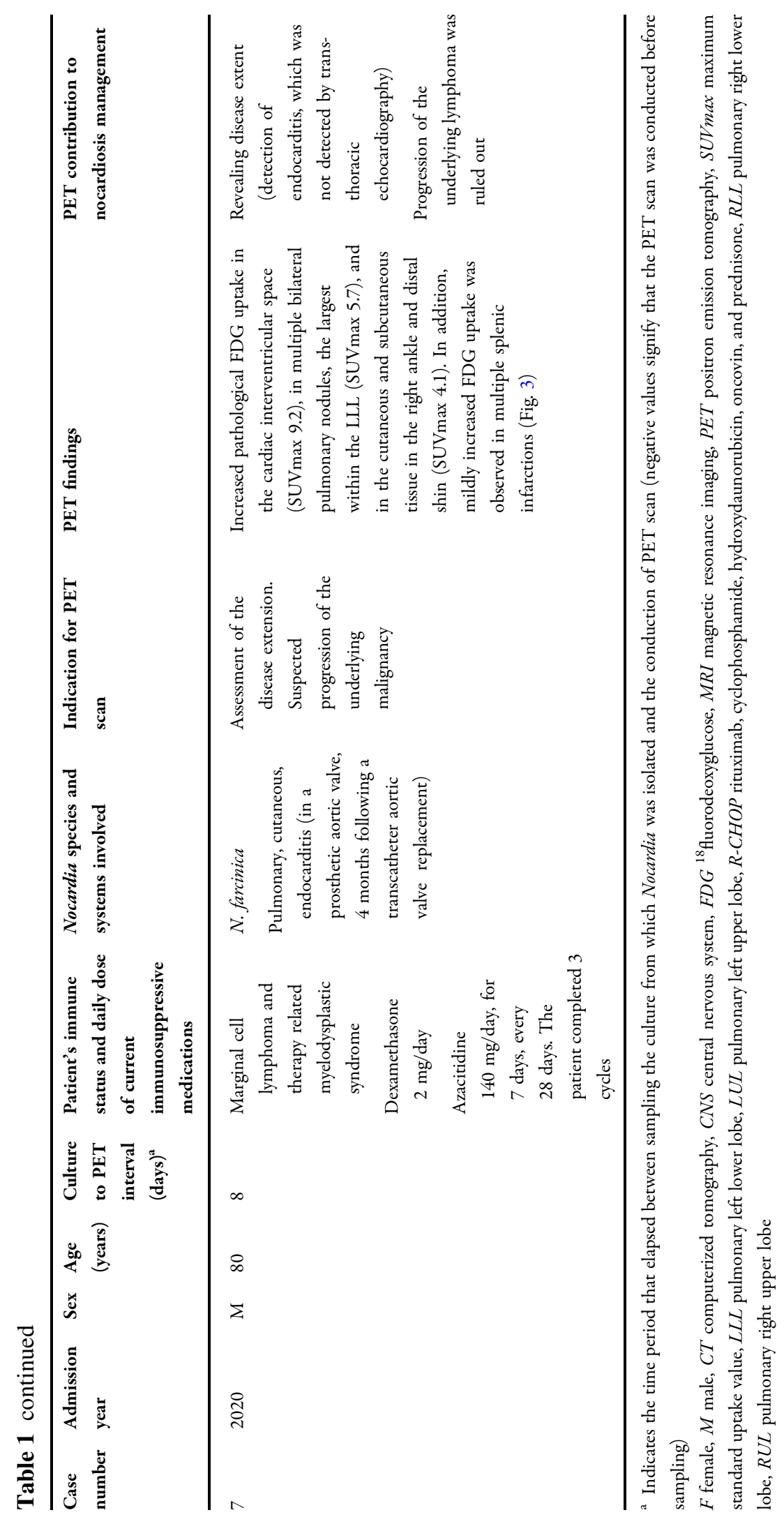



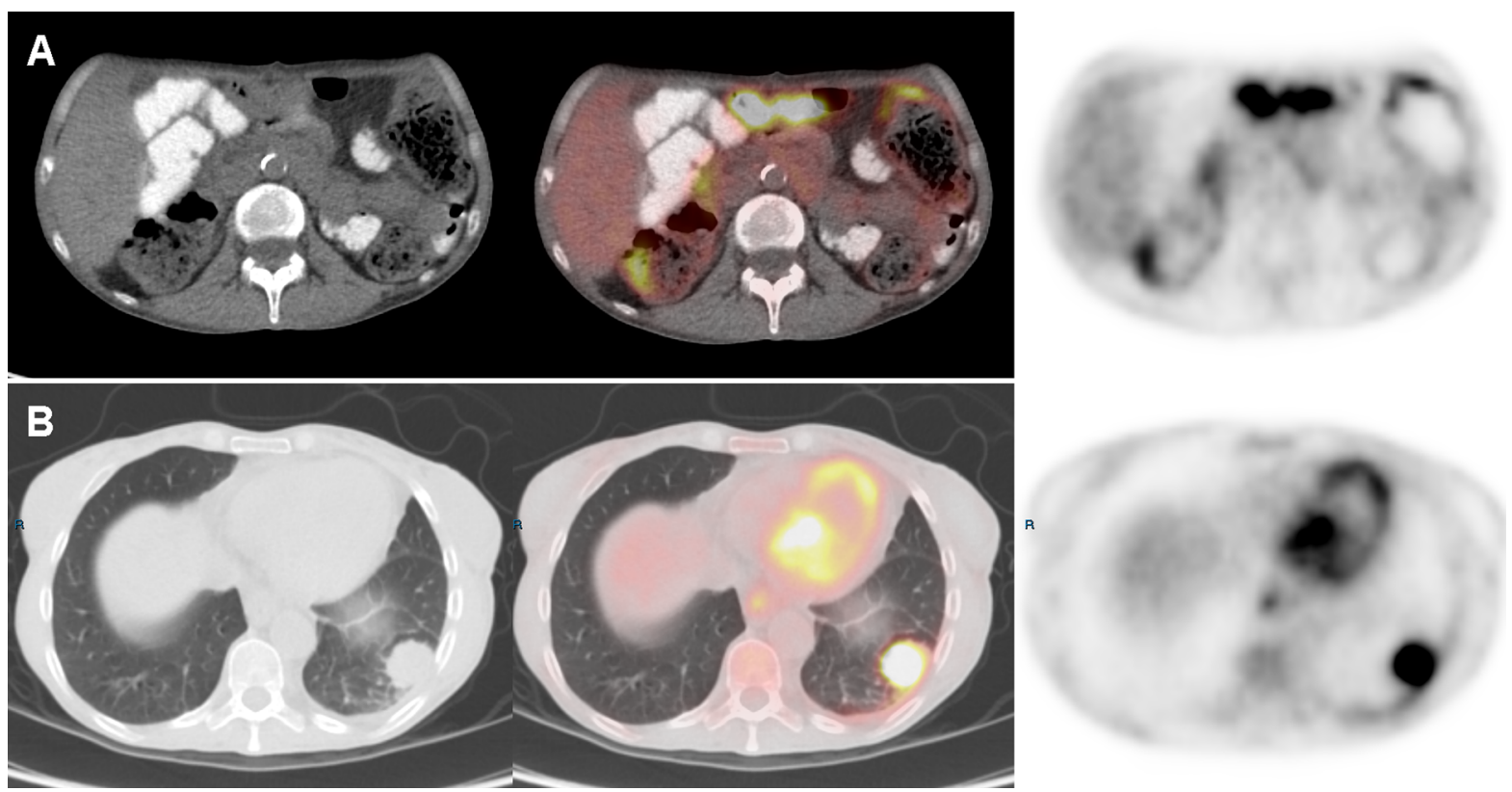

Fig. 1 Patient 3. Axial view CT, FDG-PET/CT fusion and maximum intensity projection images: a Increased pathological FDG uptake in a mass lesion in the transverse

transthoracic echocardiography could potentially guide cardiac surgery; however, the patient was frail and was therefore treated conservatively.

For the six patients for whom the FDG-PET/ CT revealed an increased pathological FDG uptake compatible with infection, the CRP level was obtained at an average time interval of 1.2 days (SD 2.2 days; range $0-6$ ) from the performance of FDG-PET/CT. The maximum SUV of the leading infectious lesion positively correlated with the CRP level $(\rho=0.77)$; however, this finding was not statistically significant $(p=0.072)$, likely because of the small sample size (Fig. 4).

\section{Systematic Literature Review}

As of October 15, 2020, the literature search yielded 105 references. These were evaluated, and a total of 19 relevant publications were identified, reporting 19 individuals with nocardiosis for whom FDG-PET/CT was used for diagnostic work-up or follow-up. An additional reference was identified through the references list of another paper. All publications were case colon, SUVmax 5.6. b Increased pathological FDG uptake in an LLL infiltrate adhesive to the pleura, SUVmax 8.1

reports of individual patients. Nine were published between 2004 and 2014 [19-27], five were published in 2015 [28-32] and six were published between 2016 and 2020 [33-38]. These 20 case reports are summarized in Table 2.

The indication for performing FDG-PET/CT was mentioned in 19/20 of the cases. The most common indication was suspected malignancy $(74 \%, 14 / 19)$, most frequently pulmonary malignancy (79\% 11/14). For the remaining five cases, the indication for performing FDG-PET/ CT was either assessment of the infection's extent $(16 \%, 3 / 19)$ or work-up for an unresolved pleural effusion, i.e., searching for an underlying inflammatory or malignant disease $(11 \%$, 2/19).

In most of these cases the performance of FDG-PET/CT contributed to the management of nocardiosis $(80 \%, 16 / 20)$. In the majority of cases $(55 \%, 11 / 20)$, the use of FDG-PET/CT guided biopsy or aspiration. FDG-PET/CT also contributed in revealing disease involvement in additional body sites, which was sometimes asymptomatic $(20 \%, 4 / 20)$, and in ruling out an underlying malignancy $(15 \%, 3 / 20)$. Contribution to treatment-related issues beyond 


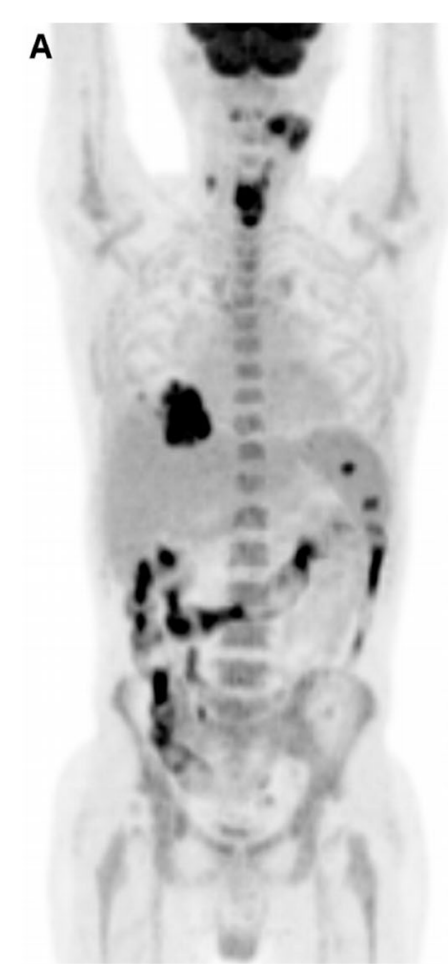

Fig. 2 Patient 4. a Anterior view maximum intensity projection showing increased pathological FDG uptake in the right lung, left neck, anterior neck, and colon. b Coronal view CT, FDG-PET/CT fusion, and maximum intensity projection images of increased pathological FDG

diagnosing a disseminated disease was not documented; however, in all reported cases FDG-PET/CT was conducted for initial work-up, usually before the diagnosis was made. In $20 \%$ $(4 / 20)$ of the cases, no contribution could be attributed to the FDG-PET/CT.

\section{DISCUSSION}

By summarizing our experience and reviewing the medical literature, we demonstrated that FDG-PET/CT may play an important role in the diagnosis and management of individuals with nocardiosis. Yet, despite its potential contribution, FDG-PET/CT is only seldom used for this purpose.

In the majority of literature cases we reviewed, FDG-PET/CT guided the location for biopsy of a suspected lesion, leading to the diagnosis of nocardiosis. In addition, FDG-PET/ CT was used as a diagnostic tool to rule out an undiagnosed underlying malignancy, a common underlying disease in nocardiosis [39].

Diagnosis of nocardiosis relies on microbiological evidence of the pathogen. Although the lungs are the most frequently affected organ [40], sputum cultures may be negative despite the existence of pulmonary infection [41]. This is particularly relevant for peripheral pulmonary or pleural infections [42]. In such cases, FDG-PET/CT can possibly guide biopsy, revealing the lesion that will most likely yield the diagnosis.

FDG-PET/CT was also frequently used for assessing extrapulmonary involvement of nocardiosis and often detected unexpected disease dissemination. We found that FDG-PET/CT identified soft tissue involvement, for which it has been reported as a more sensitive modality 


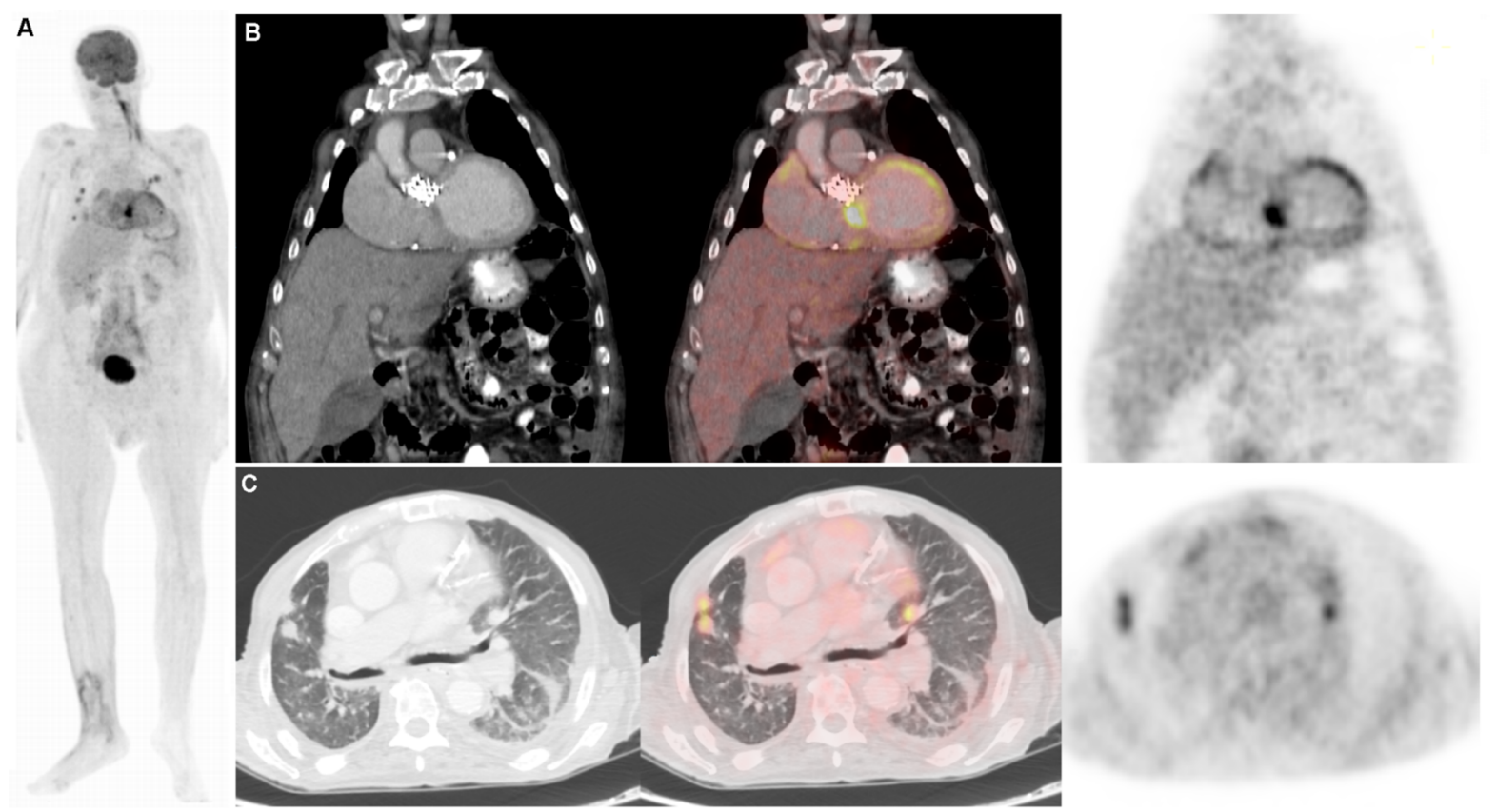

Fig. 3 Patient 7. a Anterior view maximum intensity projection showing increased pathological FDG uptake in the mediastinum, lungs, and in cutaneous and subcutaneous tissue in the right ankle and distal shin, SUVmax 4.1. In addition, increased FDG uptake of low intensity was observed in multiple splenic infarctions, SUVmax 3.3. b Coronal view CT, FDG-PET/CT fusion, and maximum intensity projection images showing increased pathological

compared to CT [43]; cardiac prosthetic material involvement that was not detected by echocardiography as reported previously [44]; and presumed abdominal involvement, not identified in the routine chest CT performed. Since most clinicians will prefer combination antibiotic therapy with longer duration for disseminated disease [18], by detecting extrapulmonary involvement, FDG-PET/CT can indirectly influence the therapeutic regimen. Nonetheless, non-specific FDG uptake does not necessarily reflect dissemination and should be interpreted with caution. This is particularly relevant for sites such as the gastrointestinal tract, an infrequent site for nocardiosis, in which incidental foci of FDG uptake were described [45]. Additionally, it should be noted that central nervous system involvement is common in nocardiosis and reported in
FDG uptake in the cardiac interventricular space, adjacent and below the transaortic inserted prosthetic valve, SUVmax 9.2. c Axial view CT, FDG-PET/CT fusion, and maximum intensity projection images showing increased pathological FDG uptake in bilateral multiple pulmonary nodules, the largest within the LLL, SUVmax 5.7; others in the LUL, SUVmax 4.8, and in the RML, SUVmax 4.7

approximately $20 \%$ of cases, usually representing disseminated disease [46]. Hence, other imaging modalities, such as magnetic resonance, are needed in addition to FDG-PET/CT. Nevertheless, our findings imply that using FDG-PET/CT routinely in cases of nocardiosis may redefine dissemination patterns of the disease and reveal a higher prevalence of dissemination than has been so far known.

Traditionally, individuals with nocardiosis are treated for long periods, usually for at least 6-12 months. Recent data suggest that much shorter durations may achieve similar cure rates [47]. Since antibiotic therapy accelerates the emergence of resistant pathogens [48] and is associated with high rates of adverse effects [49], it is imperative to shorten treatment duration whenever possible. Although none of the cases reported in the literature used FDG-PET/CT for 


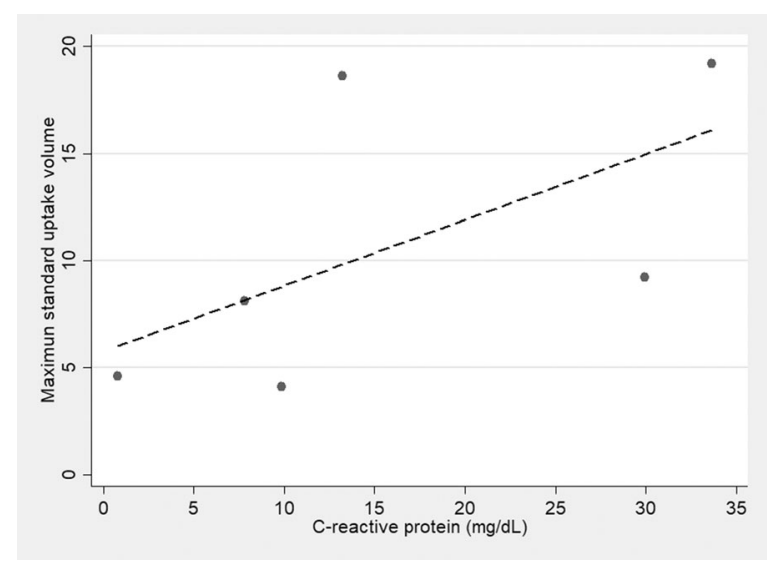

Fig. 4 A scatter plot (each dot represents an individual with nocardiosis) with fit regression line showing a possible correlation between C-reactive protein and maximum standard uptake volume of the leading lesion $(\rho=0.77$, $p=0.072$ )

therapy decision-making, it is possible that if used as an auxiliary follow-up tool it can facilitate decisions on treatment duration [50]. Similarly, FDG-PET/CT conducted shortly after the diagnosis of Staphylococcus aureus bacteremia guided treatment duration and improved survival [3]. Furthermore, FDG-PET/CT facilitated monitoring of the initial response to antibiotic therapy in infectious spondylodiscitis [51] and in predicting relapse of pulmonary tuberculosis when conducted at the time of treatment completion [52]. It is therefore possible that incorporating FDG-PET/CT at an accepted interval (e.g., 3 months from diagnosis) into the follow-up of individuals with nocardiosis may guide ongoing duration-related treatment decisions (i.e., if no pathologic FDG uptake is observed, the infection could be deemed controlled and the patient can benefit from a shorter therapeutic course).

Furthermore, our experience suggests that FDG-PET/CT can assist clinicians in other therapeutic dilemmas. We demonstrated that in the context of breakthrough fever in an individual with malignancy and nocardiosis, in which FDG-PET/CT assisted in distinguishing between antibiotic treatment failure and malignancy progression. However, although the morphology and patterns seen in CT can suggest that the FDG uptake is compatible with either malignancy or infection, the ability to differentiate between these conditions without tissue sampling is limited.

Our finding of a possible trend between CRP levels and maximum SUV is supported by a previous study on aortic graft infections, where the repertoire of pathogens does not typically include Nocardia [53]. However it seems that CRP alone cannot ultimately substitute the role of FDG-PET/CT, as treatment-related decisions are often being made according to FDG-PET/CT findings even when CRP levels are normal [53].

The current study is limited by its observational design. Accordingly, the contribution of FDG-PET/CT to the management was retrospectively appraised and is therefore subject to bias.

As for the systematic review, a publication bias is possible, i.e., cases in which FDG-PET/CT had no contribution to the management of nocardiosis were not published. Additionally, when performed, FDG-PET/CT was conducted as part of the initial management, and almost none of the included FDG-PET/CT tests were performed during follow-up. We therefore had to hypothesize about what could be the benefit of an additional FDG-PET/CT at an accepted interval.

It can be concluded that FDG-PET/CT is a potentially useful tool in the management of individuals with nocardiosis. We argue that the integration of FDG-PET/CT at baseline could add information regarding disease extent and underlying malignancies, which cannot be gained by $\mathrm{CT}$ alone. In addition, it may assist in treatment-related decisions at time of therapy initiation and during follow-up, including choice of initial and subsequent treatment regimens and duration of treatment. Further cohort study with historical control group is warranted in order to appraise the outcomes of FDG-PET/CT-guided therapy duration for individuals with nocardiosis and for determining the optimal timeframe for conducting a followup FDG-PET/CT. 


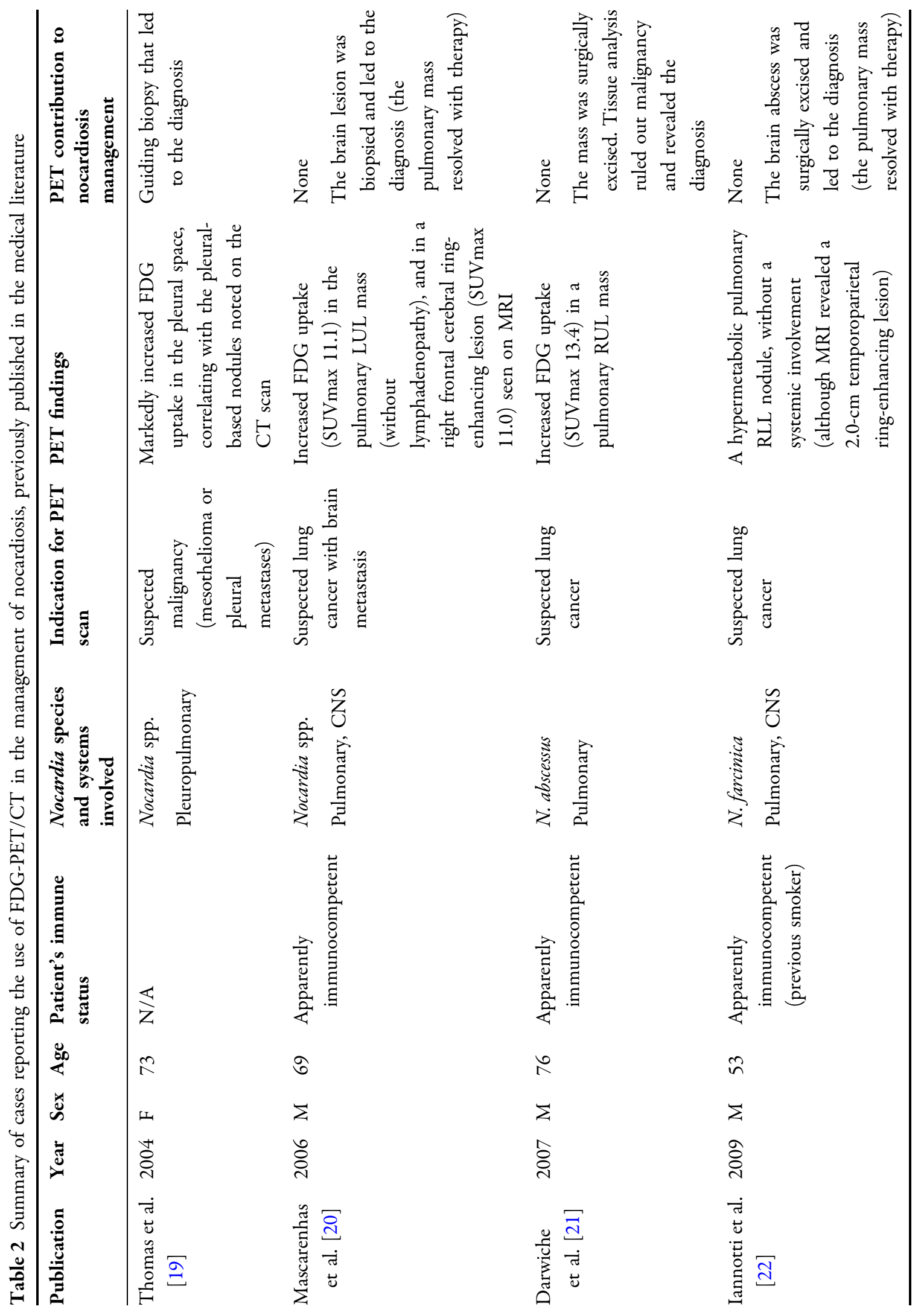




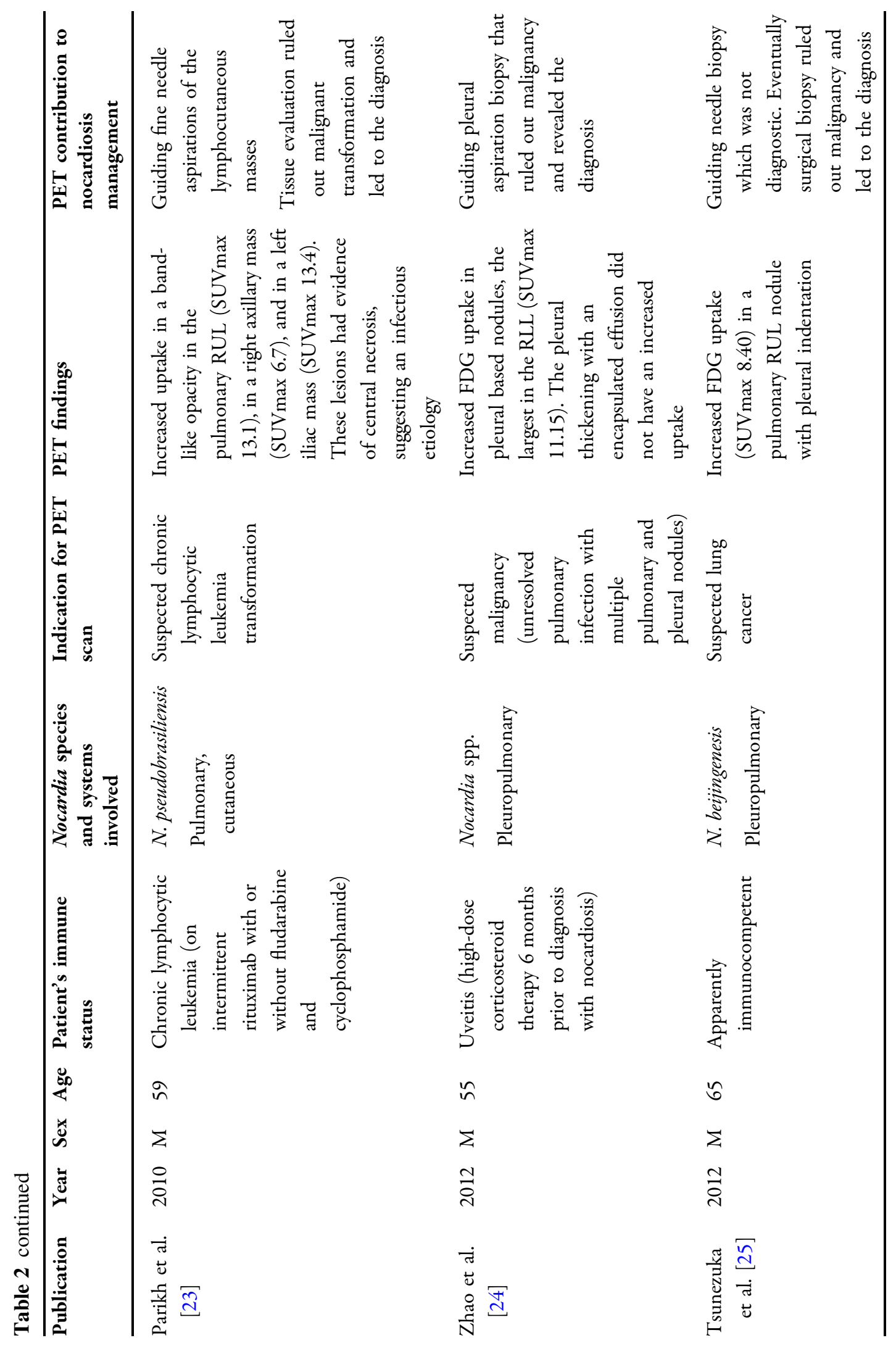




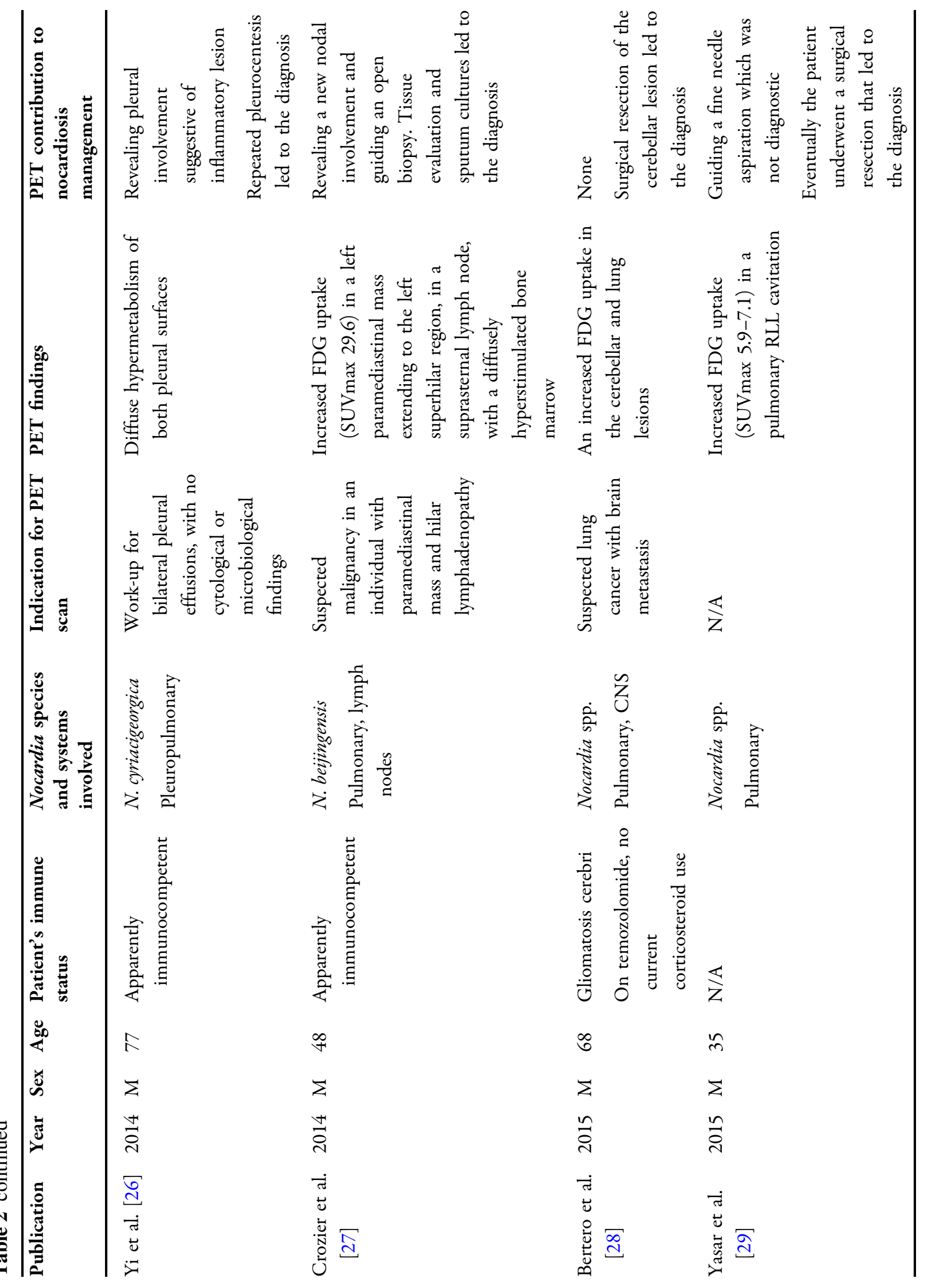




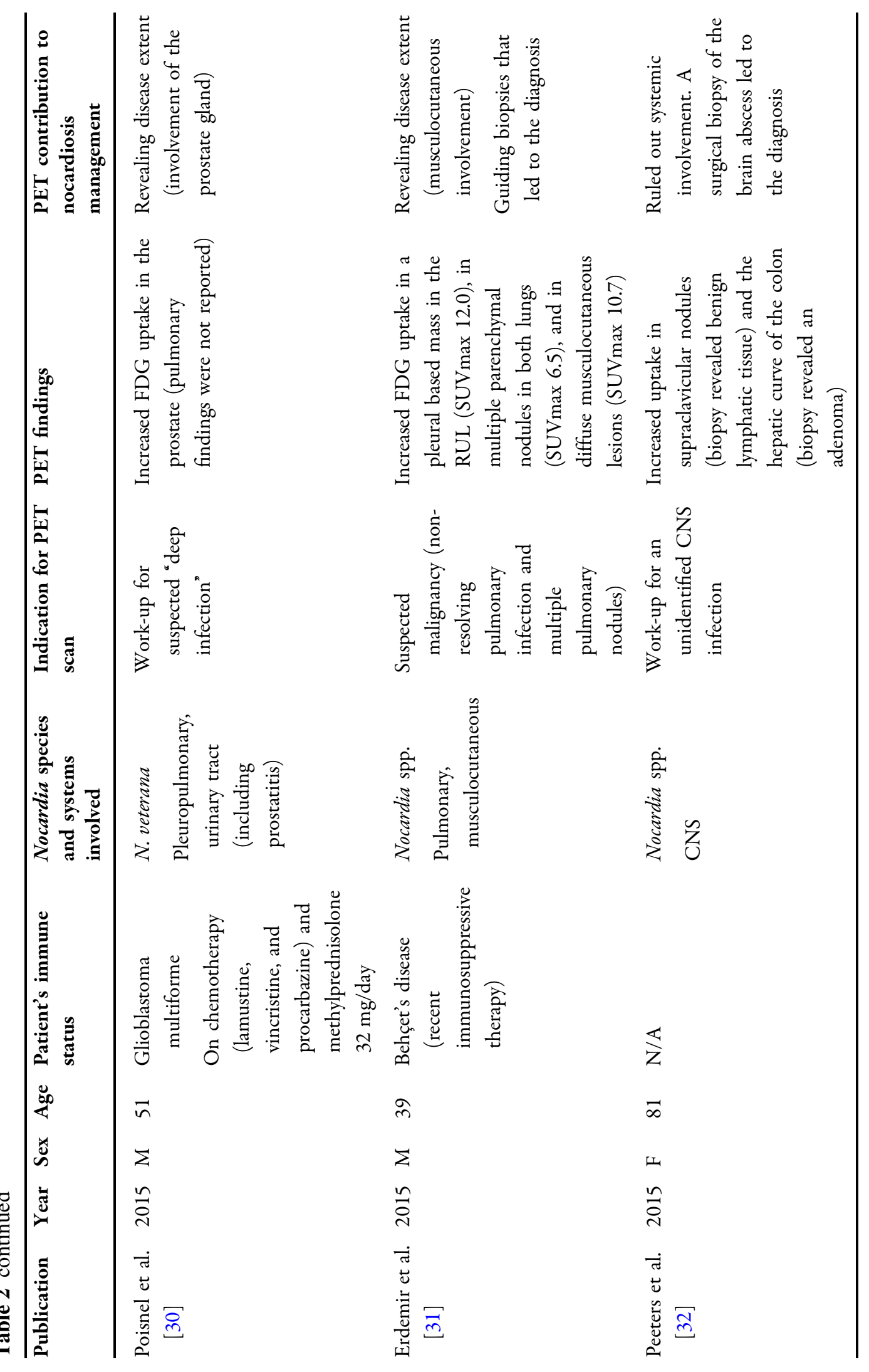




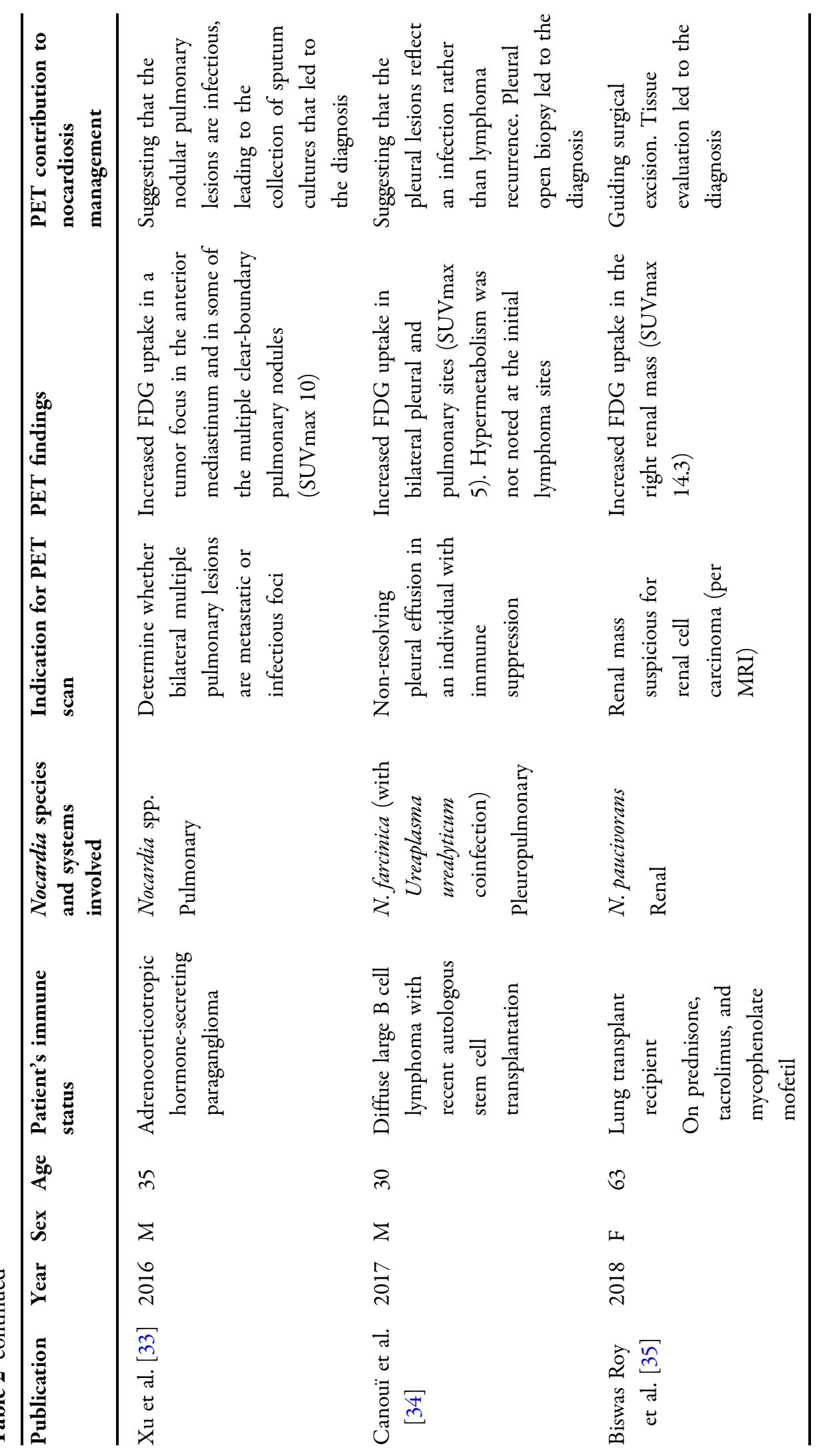




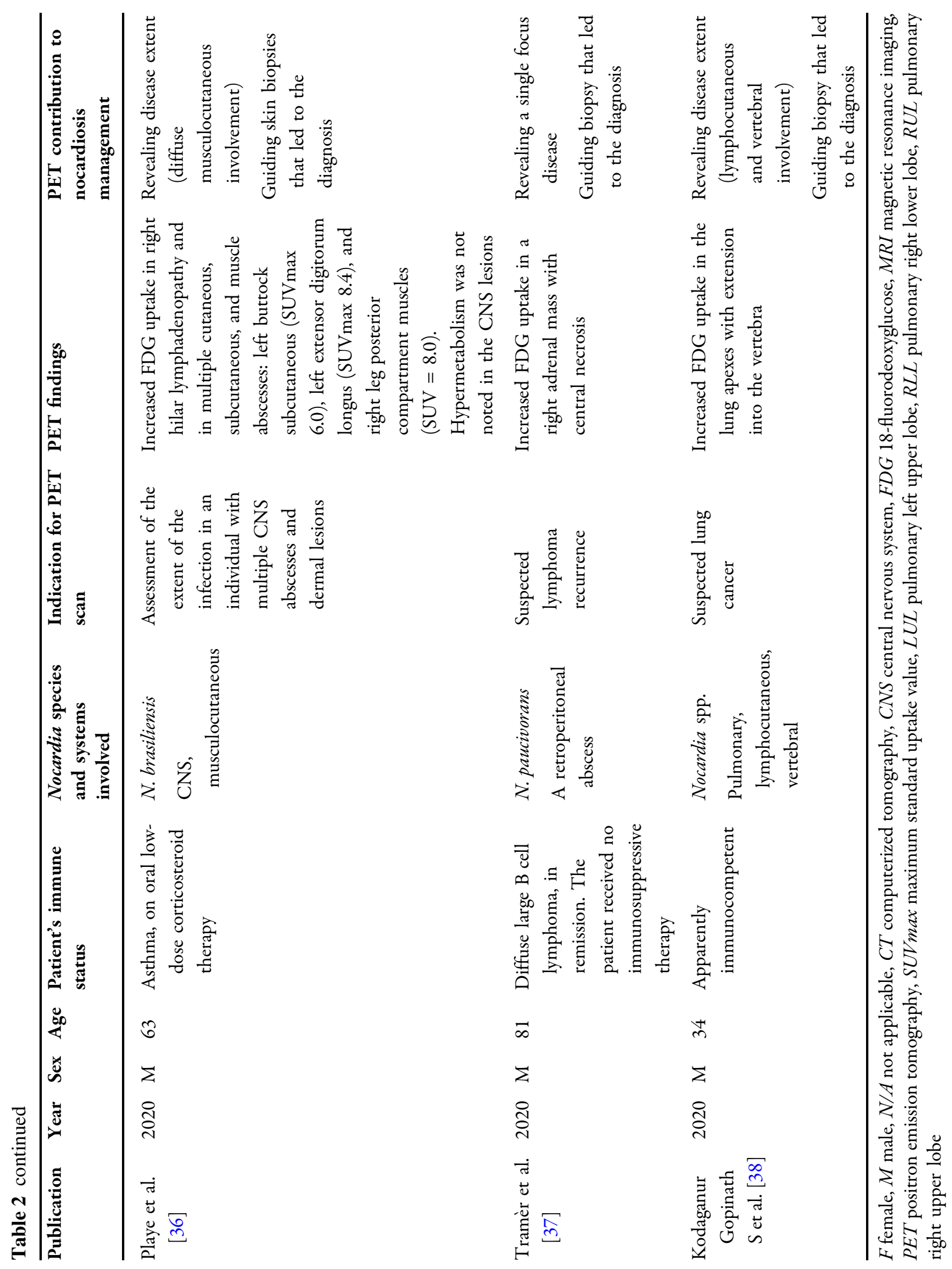




\section{ACKNOWLEDGEMENTS}

Funding. No funding or sponsorship was received for this study or publication of this article.

Authorship. All named authors meet the International Committee of Medical Journal Editors (ICMJE) criteria for authorship for this article, take responsibility for the integrity of the work as a whole, and have given their approval for this version to be published.

Author Contributions. I.M. and D.Y. conceived the study idea; I.M., E.G., J.B., and D.Y. designed the study and planned the systematic review; I.M., A.Y., Y.B.A., H.B.Z., and A.S. collected the data; I.M., M.W.S., N.T., K.M., J.B., M.P., H.B., and D.Y. drafted the manuscript; All authors contributed to manuscript revision, read and approved the submitted version.

Disclosures. Ili Margalit, Anat Yahav, Yaara Ben Ari, Haim Ben-Zvi, Avivit Shoham, Elad Goldberg, Michal Weiler-Sagie, Noam Tau, Khitam Muhsen, Jihad Bishara, Mical Paul, Hanna Bernstine, and Dafna Yahav have nothing to disclose.

Compliance with Ethics Guidelines. The ethics committees of Rabin Medical Center and Rambam Health Care Campus approved the study protocol. An exemption from informed consent was given by the ethics committees because of the retrospective design of the study. All methods were performed in accordance with the Helsinki Declaration of 1964 and its later amendments.

Open Access. This article is licensed under a Creative Commons Attribution-NonCommercial 4.0 International License, which permits any non-commercial use, sharing, adaptation, distribution and reproduction in any medium or format, as long as you give appropriate credit to the original author(s) and the source, provide a link to the Creative Commons licence, and indicate if changes were made. The images or other third party material in this article are included in the article's Creative Commons licence, unless indicated otherwise in a credit line to the material. If material is not included in the article's Creative Commons licence and your intended use is not permitted by statutory regulation or exceeds the permitted use, you will need to obtain permission directly from the copyright holder. To view a copy of this licence, visit http://creativecommons.org/licenses/by$\mathrm{nc} / 4.0 /$.

\section{REFERENCES}

1. Vaidyanathan S, Patel CN, Scarsbrook AF, Chowdhury FU. FDG PET/CT in infection and inflammation-current and emerging clinical applications. Clin Radiol. 2015;70:787-800.

2. Hess S. FDG-PET/CT in fever of unknown origin, bacteremia, and febrile neutropenia. PET Clin. 2020;15:175-85.

3. Ghanem-Zoubi N, Kagna O, Abu-Elhija J, et al. Integration of FDG-PET/CT in the diagnostic workup for staphylococcus aureus bacteremia: a prospective interventional matched-cohort study. Clin Infect Dis. 2020. https://doi.org/10.1093/cid/ ciaa929.

4. Orvin K, Goldberg E, Bernstine $\mathrm{H}$, et al. The role of FDG-PET/CT imaging in early detection of extracardiac complications of infective endocarditis. Clin Microbiol Infect. 2015;21:69-76.

5. San S, Ravis E, Tessonier L, et al. Prognostic value of 18F-fluorodeoxyglucose positron emission tomography/computed tomography in infective endocarditis. J Am Coll Cardiol. 2019;74:1031-40.

6. Erba PA, Lancellotti P, Vilacosta I, et al. Recommendations on nuclear and multimodality imaging in IE and CIED infections. Eur J Nucl Med Mol Imaging. 2018;45:1795-815.

7. Habib G, Erba PA, Iung B, et al. Clinical presentation, aetiology and outcome of infective endocarditis. Results of the ESC-EORP EURO-ENDO (European infective endocarditis) registry: a prospective cohort study. Eur Heart J. 2019;40: 3222-32.

8. Love C, Palestro CJ. Nuclear medicine imaging of bone infections. Clin Radiol. 2016;71:632-46. 
9. Reid AB, Chen SC-A, Worth LJ. Pneumocystis jirovecii pneumonia in non-HIV-infected patients. Curr Opin Infect Dis. 2011;24:534-44.

10. Sánchez-Montalvá A, Barios M, Salvador F, et al. Usefulness of FDG PET/CT in the management of tuberculosis. PLoS One. 2019;14:e0221516.

11. Leroy-Freschini B, Treglia G, Argemi X, et al. 18FFDG PET/CT for invasive fungal infection in immunocompromised patients. QJM An Int J Med. 2018;111:613-22.

12. Coussement J, Lebeaux D, van Delden C, et al. Nocardia infection in solid organ transplant recipients: a multicenter European case-control study. Clin Infect Dis. 2016;63:338-45.

13. Margalit I, Goldberg E, Ben Ari Y, et al. Clinical correlates of nocardiosis. Sci Rep Nat Res. 2020;10: 14272.

14. Garcia-Bellmunt L, Sibila O, Solanes I, Sanchez-Reus F, Plaza V. Nocardiosis pulmonar en pacientes con EPOC: características y factores pronósticos. Arch Bronconeumol. 2012;48:280-5.

15. Wang HL, Seo YH, LaSala PR, Tarrand JJ, Han XY. Nocardiosis in 132 patients with cancer. Am J Clin Pathol. 2014;142:513-23.

16. Fatahi-Bafghi M. Nocardiosis from 1888 to 2017. Microb Pathog. 2018;114:369-84.

17. Margalit I, Muhsen K, Ben Ari Y, et al. Nocardia colonization in contrast to nocardiosis: a comparison of patients' clinical characteristics. Eur J Clin Microbiol Infect Dis. 2020;39:759-63.

18. Restrepo A, Clark NM. Infectious Diseases Community of Practice of the American Society of Transplantation. Nocardia infections in solid organ transplantation: Guidelines from the Infectious Diseases Community of Practice of the American Society of Transplantation. Clin Transpl. 2019;1: e13509.

19. Thomas A, Singh A, Greenspan B. Localization of F-18 FDG in Nocardia lung infection. Clin Nucl Med. 2004;29:834-5.

20. Mascarenhas NB, Lam D, Lynch GR, Fisher RE. PET imaging of cerebral and pulmonary Nocardia infection. Clin Nucl Med. 2006;31:131-3.

21. Darwiche K, Totzeck B, Gatermann S, Bollow M, Ewig S. Nocardiosis as a differential diagnosis of a PET-positive pulmonary nodule. Pneumologie. 2007;61:176-80.

22. Iannotti CA, Hall GS, Procop GW, Tuohy MJ, Staugaitis SM, Weil RJ. Solitary Nocardia farcinica brain abscess in an immunocompetent adult mimicking metastatic brain tumor: rapid diagnosis by pyrosequencing and successful treatment. Surg Neurol. 2009;72:74-9.

23. Parikh SA, Bhusal Y, Faderl SH, et al. The great imitator: systemic nocardiosis mimicking Richter's transformation in relapsed chronic lymphocytic leukemia. J Clin Oncol. 2010;28:e732-4.

24. Zhao K, Dong M-J, Sheng Z-K, et al. Elevated uptake of 18F-FDG in PET/CT imaging of a Nocardial pleural nodule. Clin Imaging. 2012;36:383-5.

25. Tsunezuka Y, Furusawa T, Fujimori H, Kurumaya H. Pulmonary nocardiosis with a tumor-like solitary nodule caused by Nocardia beijingenesis: first report of human surgical infection. Surg Infect (Larchmt). 2012;13:418-9.

26. Yi C, Kwon MJ, Ki CS, et al. Necrotizing pneumonia and empyema in an immunocompetent patient caused by Nocardia cyriacigeorgica and identified by 16S rRNA and secA1 sequencing. Ann Lab Med. 2014;34:71.

27. Crozier JA, Andhavarapu S, Brumble LM, Sher T. First report of Nocardia beijingensis infection in an immunocompetent host in the United States. J Clin Microbiol. 2014;52:2730-2.

28. Bertero L, Cassoni P, Rudà R. Nocardia abscesses mimicking tumor progression in gliomatosis cerebri responding to temozolomide. J Neurooncol. $2015 ; 121: 225-7$.

29. Yasar Z, Acat M, Onaran H, et al. False-positive 18-fluorodeoxyglucose positron emission tomography-computed tomography (FDG PET/CT) scans mimicking malignancies. Med Glas (Zenica). 2015;12:40-6.

30. Poisnel E, Roseau JB, Landais C, Rodriguez-Nava V, Bussy E, Gaillard T. Nocardia veterana: disseminated infection with urinary tract infection. Braz J Infect Dis. $2015 ; 19: 216-9$.

31. Erdemir RU, Elri T, Sahin H, Sami Salihoglu Y, Aras M, Cabuk M. Disseminated Nocardia infection mimicking malignancy on FDG PET/CT. Rev Esp Med Nucl Imagen Mol. 2015;34:268-9.

32. Peeters I, Casselman JW, Vandecasteele SJ, et al. Multiple Nocardial abscesses of the brainstem and spinal cord diagnosed after an open biopsy through a cervical partial central corpectomy: case report. J Neurosurg Spine. 2015;23:290-3.

33. Xu L, Xu Q, Yang M, Gao H, Xu M, Ma W. Nocardiosis in ectopic ACTH syndrome: a case report and review of 11 cases from the literature. Exp Ther Med. 2016;12:3626-32. 
34. Canouï E, Blanc K, Loubinoux J, et al. The value of molecular techniques to diagnose Ureaplasma urealyticum and Nocardia farcinica pleuropneumonia in a patient with diffuse large B-cell lymphoma. Int J Infect Dis. 2017;64:93-5.

35. Biswas Roy S, Ross MD, Patil PD, Trepeta R, Bremner RM, Panchabhai TS. Primary Nocardia infection causing a fluorodeoxyglucose-avid right renal mass in a redo lung transplant recipient. Case Rep Transpl. 2018;2018:1-5.

36. Playe M, Einfalt M, Toch SR, Froissart A, Bodardel G. 18F-FDG imaging of a case of disseminated nocardiosis. Clin Nucl Med. 2020;45:55-6.

37. Tramèr L, Mertz K, Huegli R, et al. Intra-abdominal nocardiosis-case report and review of the literature. J Clin Med. 2020;9:2141.

38. Kodaganur Gopinath S, Pulle MV, Dhamija A, Puri $\mathrm{H}$, Bin Asaf B, Kumar A. A rare disease mimicking lung cancer. Indian J Tuberc Tuberculosis Association of India. 2020;67:430-2.

39. Robin P, van Es N, Le Roux PY, et al. Performance of 18F-fluorodesoxyglucose positron-emission tomography/computed tomography for cancer screening in patients with unprovoked venous thromboembolism: results from an individual patient data meta-analysis. Thromb Res Elsevier Ltd. 2020;194: 153-7.

40. Minero MV, Marín M, Cercenado E, Rabadán PM, Bouza E, Muñoz P. Nocardiosis at the turn of the century. Medicine (Baltimore). 2009;88:250-61.

41. Li S, Song X, Zhao Y, et al. Clinical analysis of pulmonary nocardiosis in patients with autoimmune disease. Medicine (Baltimore). 2015;94: e1561.

42. Sukahri S, Zainudin LD, Hadi MF, Mohd Firdaus MAB, Abdul Hafidz MI. Recurrent empyema thoracic secondary to pulmonary nocardiosis in immunocompetent patients. Case Rep Pulmonol. 2020;2020:8840920. https://doi.org/10.1155/2020/ 8840920 .

43. Palestro CJ. FDG-PET in musculoskeletal infections. Semin Nucl Med. 2013;43:367-76.

44. Chen W, Kim J, Molchanova-Cook OP, Dilsizian V. The potential of FDG PET/CT for early diagnosis of cardiac device and prosthetic valve infection before morphologic damages ensue. Curr Cardiol Rep. 2014;1:16.

45. Israel O, Yefremov N, Bar-Shalom R, et al. PET/CT detection of unexpected gastrointestinal foci of 18F-FDG uptake: incidence, localization patterns, and clinical significance. J Nucl Med. 2005;46: 758-62.

46. Beaman BL, Beaman L. Nocardia species: host-parasite relationships. Clin Microbiol Rev. 1994;7: 213-64.

47. Lebeaux D, Freund R, van Delden C, et al. Outcome and treatment of nocardiosis after solid organ transplantation: new insights from a European study. Clin Infect Dis. 2017;64:1396-405.

48. Davies J, Davies D. Origins and evolution of antibiotic resistance. Microbiol Mol Biol Rev. 2010;74:417-33.

49. Tamma PD, Avdic E, Li DX, Dzintars K, Cosgrove SE. Association of adverse events with antibiotic use in hospitalized patients. JAMA Int Med. 2017;177: 1308-15.

50. Stern Shavit S, Bernstine H, Sopov V, Nageris B, Hilly O. FDG-PET/CT for diagnosis and follow-up of necrotizing (malignant) external otitis. Laryngoscope. 2019;129:961-6.

51. Righi E, Carnelutti A, Muser D, et al. Incremental value of FDG-PET/CT to monitor treatment response in infectious spondylodiscitis. Skeletal Radiol. 2020;49:903-12.

52. Lawal IO, Fourie BP, Mathebula M, et al. 18F-FDG PET/CT as a noninvasive biomarker for assessing adequacy of treatment and predicting relapse in patients treated for pulmonary tuberculosis. J Nucl Med. 2020;61:412-7.

53. Husmann L, Ledergerber B, Anagnostopoulos A, et al. The role of FDG PET/CT in therapy control of aortic graft infection. Eur J Nucl Med Mol Imaging. 2018;45:1987-97.

\section{Publisher's Note}

Springer Nature remains neutral with regard to jurisdictional claims in published maps and institutional affiliations. 